\title{
Collaborative work on evaluation of ovarian toxicity 12) Effects of 2- or 4-week repeated dose studies and fertility study of indomethacin in female rats
}

\author{
Kenjiro Tsubota, Kiyoshi Kushima, Kenji Yamauchi, Seiki Matsuo, Tadashi Saegusa, \\ Shin Ito, Michio Fujiwara, Masahiro Matsumoto, Shunji Nakatsuji, Jiro Seki and Yuji Oishi \\ Toxicologic Pathology, Drug Safety Research Laboratories, Astellas Pharma Inc., 1-6, Kashima 2-chome, \\ Yodogawa-ku, Osaka 532-8514, Japan
}

(Received December 10, 2008)

\begin{abstract}
- 2-week and 4-week general toxicity studies of indomethacin, a nonselective inhibitor of cyclooxygenase 1 and 2, were performed using rats. A female fertility study was also conducted to compare the results to those of ovarian histopathological findings. The main purposes of the present studies are to assess whether a precise histopathological examination, taking the morphological changes the female reproductive organs undergo during each estrus phases into account, can evaluate toxicity to the ovaries, and to determine the optimal administration period for detecting ovarian toxicity. Indomethacin was administered on a daily basis to female Sprague-Dawley rats at doses of $0,0.4,1.3$, or $4 \mathrm{mg} / \mathrm{kg}$ in the both the general toxicity studies and the female fertility study. In the general toxicity studies, unruptured follicles or luteinized cysts were observed histopathologically in the $4 \mathrm{mg} / \mathrm{kg}$ group in both the 2-week and 4week studies. In addition, follicular cysts were found in the $4 \mathrm{mg} / \mathrm{kg}$ group in the 4-week study. Estrous cyclicity was not disturbed in both studies. There were no histopathological changes in the ovaries of the $1.3 \mathrm{mg} / \mathrm{kg}$ group in general toxicity studies. In the female fertility study, no toxic effects on female fertility parameters were detected in the 0.4 and $1.3 \mathrm{mg} / \mathrm{kg}$ group treated with indomethacin, but 8 of 10 rats in the $4 \mathrm{mg} / \mathrm{kg}$ group died or were sacrificed before completion of the dosing period. These results demonstrated that 2 weeks of indomethacin treatment is sufficient to detect unruptured follicles or luteinized cyst in the ovary. In addition, 4 weeks of dosing maybe required for induction of follicular cysts, although we could not clearly show that these histopathological changes would affect female fertility functions. These present studies suggest that a precise histopathological examination may be able to predict the effect of test articles on female reproductive functions.
\end{abstract}

Key words: Indomethacin, Reproductive toxicity, Ovary, Fertility, Rat

\section{INTRODUCTION}

According to current ICH M3 guidelines, the completion of a female fertility study is required prior to the enrollment of women of childbearing potential in any clinical trials conducted in Japan. In the USA and the $\mathrm{EU}$, this is required prior to phase III clinical trials only. To harmonize these regional differences, collaboration among the members of the Japan Pharmaceutical Manufacturers Association was organized to conduct validation studies.

Indomethacin is a well-known nonselective inhibitor of cyclooxygenase (COX)-1 and -2 , which are involved in prostaglandin synthesis from arachidonic acid. This sub- stance is used worldwide as a non-steroidal anti-inflammatory drug (NSAID) and an arylalkanoic acid class analgesic (Brogden, 1986). Many non-clinical toxicity studies have clearly shown NSAID-induced gastrointestinal damage or renal papillary necrosis (Khan et al., 1998; Satoh et al., 1981). In addition, indomethacin has been thought to inhibit ovulation by acting directly and specifically on the preovulatory follicles of rat ovaries, where prostaglandin synthesis is markedly increased (Sato et al., 1974; Tsafriri et al., 1972; Armstrong and Grinwich, 1972).

Taking the regulation of female sex hormones, morphological changes in the ovary and other reproductive organs during each estrus phase into account, ovarian toxicity may be precisely evaluated via histopathological

Correspondence: Kenjiro Tsubota (E-mail: kenjiro.tsubota@jp.astellas.com) 
examination of the female reproductive organs. To validate the use of a such a general toxicity study for prediction of toxic effects on female reproductive functions and to optimize the appropriate administration period, 2and 4-week repeated dose general toxicity studies and a female fertility study were conducted.

\section{MATERIALS AND METHODS}

\section{Test article}

Indomethacin was obtained from Sigma-Aldrich (Sigma-Aldrich, St. Louis, MO, USA), and suspended in $0.5 \%$ methylcellulose solution $\left(\right.$ Metlose $^{\circledR}, \mathrm{SM}-400$, Shin-Etsu Chemical Co., Ltd., Tokyo, Japan) in concentrations of $0.08,0.26$, and $0.8 \mathrm{mg} / \mathrm{ml}$. The dose levels for the general toxicity studies and the female fertility study were selected according to the results of a preliminary study performed on 5 female rats receiving $3 \mathrm{mg} / \mathrm{kg}$ of indomethacin by gavage for 15-days. Decreased body weight, slight peritonitis, and an enlarged spleen were observed in one animal. The highest dose for the general toxicity studies and the fertility study were set at $4.0 \mathrm{mg} /$ $\mathrm{kg}$ in order to determine the more severe toxic effects of indomethacin. The dose level was divided using a common ratio of 3 to determine the intermediate and low doses of 1.3 and $0.4 \mathrm{mg} / \mathrm{kg}$, respectively. A group of rats receiving only vehicle served as the control in each study.

\section{Animals}

For the general toxicity studies, 5-week-old female Crl: $\mathrm{CD}(\mathrm{SD})$ rats were purchased from Charles River Japan, Inc. (Charles River Japan, Kanagawa, Japan). After 1week of acclimatization, the 6-week-old animals, weighing 148-174 g, were used. For the fertility study, 9-weekold male and female $\mathrm{Crl}: \mathrm{CD}(\mathrm{SD})$ rats were purchased. Female rats weighing 225-228 g after 1-week of acclimatization and male rats weighing an average of $413.6 \mathrm{~g}$ at 12 weeks of age were used for the study.

Two animals were housed in each metal cage in a barrier room with a specific pathogen-free environment, a temperature of 22.4 to $23.8^{\circ} \mathrm{C}$, a relative humidity of 45 to $62 \%$, and a 12 -hr light-dark cycle. A standard commercial diet (CRF-1, Oriental Yeast Co., Ltd., Tokyo, Japan) and tap water were available ad libitum. The animals were weighed on the day of treatment initiation, and then allocated to groups using a computerized randomization process based on body weight. During the study, animals were handled in accordance with Guideline for Animal Experimentation issued by Astellas Pharma Inc., which is based on the Guideline for Animal Experimentation (Japanese Association for Laboratory Animal Science, 1987).

\section{Study Designs}

\section{General toxicity studies}

Indomethacin was orally administered to female rats at dose levels of $0.4,1.3$, and $4 \mathrm{mg} / \mathrm{kg}$ for 2 (2-week study) or 4 weeks (4-week study) (10 animal/ group). All animals were observed for general signs 3 times a day (once before dosing and twice after dosing) during the dosing period. Body weights and food intake were recorded for each cage twice a week during the dosing period. Estrus phases were determined for each animal by examining vaginal smears every day for last 7 and 14 days during the 2- and 4-week studies, respectively, before completion of dosing. At the end of the dosing period, the animals were sacrificed by exsanguination under anesthesia. Blood samples were collected for measuring the coagulation and hematological parameters and for blood chemistry testing. The ovaries, uterus, vagina and pituitary were weighed and fixed in $10 \%$ neutral buffered formalin. These organs were embedded in paraffin, sectioned at $4 \mu \mathrm{m}$, and stained with hematoxylin and eosin (HE), according to routine procedure. At the time of section preparation, both ovaries were transversally halved for observation of the maximum area. In addition to HE staining, the ovarian sections were subjected to immunohistochemical staining with mouse anti-proliferating cell nuclear antigen monoclonal antibody (antiPCNA mAb, Dako Cytomation, Glostrup, Denmark, code No. M0879). Histofine ${ }^{\circledR}$ Simple Stain Rat MAX-PO (MULTI) (Nichirei, Tokyo, Japan) was used as the second antibody, and Histofine ${ }^{\circledR}$ Simple Stain DAB solution (Nichirei) was used to visualize the proliferative cell nuclear antigen (PCNA). Specimens of the heart, spleen, stomach, duodenum, jejunum ileum, cecum, colon, rectum, liver, kidney, adrenal, mammary gland, and organs in which abnormalities were found at necropsy were also prepared. The dead animals were necropsied as soon as they were found, after which their organs were fixed, and specimens prepared. These specimens were examined microscopically. In the ovary, HE-stained and anti-PCNA stained specimens were examined, and their abnormalities evaluated comprehensively.

\section{Female fertility study}

Indomethacin was orally administered to female rats at dose levels of $0,0.4,1.3$, and $4.0 \mathrm{mg} / \mathrm{kg}$ (10 animals/ group). The daily dosing of indomethacin or vehicle continued for 2 weeks before the mating period, through the mating period, and until the 7 th day of pregnancy. Animals were necropsied on the 14th day of pregnancy, and the thoracic and intraperitoneal organs were examined 
Repeated dose toxicity and fertility studies of indomethacin in rats

macroscopically. The number of corpora lutea, implantations, and live and dead embryos were counted, and the conception and pregnancy rates were calculated. Vaginal smears were also collected from all females daily for 14 days before cohabitation to determine the timing of the estrus phase.

\section{Statistical analyses}

Body weight, food intake, hematology, blood chemistry, organ weight (absolute and relative), frequency of the estrus phase, and the numbers of corpora lutea, implantations, live fetuses, embryo-fetal deaths, and days until copulation were analyzed statistically. Homogeneity of variances was analyzed using Bartlett's test at $\mathrm{P}<0.01$ (two-tailed test). When a set of variances was homogenous, the treatment and control group values were analyzed using Dunnett's multiple comparison test (Dunnett, 1964) at $\mathrm{P}<0.05$ or $\mathrm{P}<0.01$ (two-tailed test). When a set of variances was not homogenous, the parameters were first rank-transformed, and then analyzed in the same way as a homogenous case.

Wilcoxon's rank-sum test (Mann-Whitney's U test) was applied to pre- and post-implantation loss in the fertility study.

\section{RESULTS}

\section{General toxicity studies}

In the 2-week study, no abnormal clinical signs were observed in any groups, including the control, throughout the treatment period and at necropsy (Table 1). In the 4-week study, 2 animals in the $4 \mathrm{mg} / \mathrm{kg}$ group showed a decrease in food consumption and suppression of body weight gain. These animals were found dead on the $22 \mathrm{nd}$ and 24 th day of the dosing period (Table 2) because of peritonitis due to gastrointestinal ulcers. One of the surviving animals in the $4 \mathrm{mg} / \mathrm{kg}$ group showed the same clinical signs and severe peritonitis upon terminal necropsy. A hard focus was noted in the jejunum and ileum of the other 2 surviving animals in the $4 \mathrm{mg} / \mathrm{kg}$ group. Any

Table 1. Summary of clinical observations in 2-week study

\begin{tabular}{lcccc}
\hline Dose (mg/kg) & 0 & 0.4 & 1.3 & 4 \\
\hline No. of Animals & 10 & 10 & 10 & 10 \\
No. of Animals Died or Sacrificed Moribund & 0 & 0 & 0 & 0 \\
Clinical Observations & - & - & - & - \\
Body Weight (g): Day 15 (necropsy day) & 186 & 188 & 186 & 188 \\
Body Weight Gain (g/14 days) & 39 & 39 & 36 & 41 \\
Food Consumption (g/day/animal) & 17 & 17 & 17 & 17 \\
Mean Estrous Cycle (days) & 4 & 4 & 4 & 4 \\
No of irregular estrous cycles ${ }^{1)}$ & $0 / 10$ & $0 / 10$ & $0 / 10$ & $0 / 10$ \\
\hline
\end{tabular}

1) Irregular cycle was defined as having an interval between estrus phases of longer than 4 days, or being anestrous,

-: No noteworthy findings.

$*: \mathrm{p}<0.05, * *: \mathrm{p}<0.01$

Table 2. Summary of clinical observations in 4-week study

\begin{tabular}{|c|c|c|c|c|}
\hline Dose $(\mathrm{mg} / \mathrm{kg})$ & 0 & 0.4 & 1.3 & 4 \\
\hline No. of Animals & 10 & 10 & 10 & 10 \\
\hline No. of Animals Died or Sacrificed Moribund & 0 & 0 & 0 & 2 \\
\hline Clinical Observations & - & - & - & \\
\hline Moist fur around urethral orifice & & & & 3 \\
\hline Decreased spontaneous motility & & & & 2 \\
\hline Body Weight (g): Day 29 (necropsy day) & 217 & 217 & 219 & 208 \\
\hline Body Weight Gain ( $\mathrm{g} / 28$ days) & 79 & 77 & 76 & 65 \\
\hline Food Consumption (g/day/animal) & 18 & 18 & 18 & 17 \\
\hline Mean Estrous Cycle (day) & 4 & 4 & 4 & 4 \\
\hline No of irregular estrous cycle ${ }^{1)}$ & $0 / 10$ & $0 / 10$ & $0 / 10$ & $1 / 10$ \\
\hline
\end{tabular}

1) Irregular cycle was defined as having an interval between estrus phases of longer than 4 days, or being anestrous,

-: No noteworthy findings.

$*: \mathrm{p}<0.05, * *: \mathrm{p}<0.01$ 
disturbance was not detected in estrous cyclicity in both of the studies.

In the 2-week study, the hematology test results showed a decrease in hemoglobin and increases in the reticulocyte ratio, platelet count, and fibrinogen concentration at $4 \mathrm{mg} / \mathrm{kg}$. In the 4-week study, decreases in the hematocrit value, hemoglobin concentration, red blood cell count, and mean corpuscular hemoglobin concentration as well as increases in the reticulocyte ratio, neutrophil count and fibrinogen concentration were observed in the $4 \mathrm{mg} / \mathrm{kg}$ group. Blood chemistry testing for the 2 -week study showed decreases in total protein and albumin and increases in chloride were observed in the $4 \mathrm{mg} / \mathrm{kg}$ group. The total protein and albumin concentrations and albu$\mathrm{min} /$ globulin ratios in the 4-week study decreased in the $4 \mathrm{mg} / \mathrm{kg}$ group, but the chloride concentration increased. These changes in hematology and blood chemistry may be related to anemia or inflammation and peritonitis secondary to ulcers.

No significant changes in mean organ weight were observed for the ovary, uterus or pituitary (Tables 3 and 4). In the other organs, increases in the absolute and relative weight of the spleen were observed in the $4 \mathrm{mg} /$ $\mathrm{kg}$ group of the 2-week study. Increases in the absolute weights of the spleen and liver were also observed in the $4 \mathrm{mg} / \mathrm{kg}$ group of the 4-week study.

Histopathological findings for the ovary are summarized in Tables 5 and 6. Luteinized cyst or unruptured follicle were mixed with normal morphological changes of the ovaries in 3 and 2 animals in the $4 \mathrm{mg} / \mathrm{kg}$ groups of the 2- and 4-week studies, respectively. In the 2-week study, an ovum remained in the cavity of a luteinized cyst in an animal which showed the metestrus phase. A cavity in the luteinized cyst was not so large, and the luteal cells showed the same morphology as those in normal corpus luteum at metestrus (Fig. 1). All other animals were in the estrus phase. Unruptured follicles in the estrus phase were large and cystic, and the luteinized granulosa cells, increased mitotic figures and proliferation of the blood vesselswere observed. In the 4-week study an ovum surrounded by expanded oophorus cumulus was seen in the unruptured follicle in one of the animals (Fig. 2). In addition, follicular cysts (Fig. 3) were observed in one ani$\mathrm{mal}$ in the $4 \mathrm{mg} / \mathrm{kg}$ group in the 4 -week study. The cystic follicles found on both sides of the ovary were much larger than large-sized atretic follcles, and apoptotic figures of granulosa cells were more prominent. There were no abnormal findings in pituitary, uterus, and in vagina in both studies.

Ulcer, focal peritonitis, submucosal inflammatory cell infiltration of the cecum, and increased extramedullary hematopoiesis of the spleen were found in the $4 \mathrm{mg} / \mathrm{kg}$ group of the 2 -week study. In the $4 \mathrm{mg} / \mathrm{kg}$ group of the 4 week study, increased extramedullary hematopoiesis and peritonitis of the spleen, atrophy of the thymus, peritonitis of the stomach and duodenum, peritonitis and ulcer of the jejunum, peritonitis and ulcer of the ileum, ulcer and peritonitis of the cecum, increased incidences of microgranuloma, sinusoidal cell hypertrophy, extramedullary hematopoesis and peritonitis of the liver, papillary necrosis, hyaline droplets and vacuolar changes in the proximal tubules, tubular dilatation of the kidney, extramedullary hematopoiesis and presence of cortical eosinophilic bodies, and vacuolar change in the zona glomerulosa of the

Table 3. Summary of organ weights in the 2-week study

\begin{tabular}{|c|c|c|c|c|}
\hline Dose $(\mathrm{mg} / \mathrm{kg})$ & 0 & 0.4 & 1.3 & 4.0 \\
\hline No. of Animals & 10 & 10 & 10 & 10 \\
\hline Ovary (R) (mg) & $33 \pm 5.3$ & $36 \pm 7.8$ & $33 \pm 6.5$ & $33 \pm 9.0$ \\
\hline Ovary (L) (mg) & $33 \pm 8.6$ & $33 \pm 8.7$ & $38 \pm 6.4$ & $35 \pm 6.0$ \\
\hline Pituitary (mg) & $12 \pm 1.7$ & $12 \pm 1.5$ & $12 \pm 1.6$ & $12 \pm 0.8$ \\
\hline Uterus (mg) & $401 \pm 114.4$ & $441 \pm 190.7$ & $373 \pm 108.0$ & $387 \pm 80.6$ \\
\hline
\end{tabular}

$*: \mathrm{p}<0.05, * *: \mathrm{p}<0.01$

Table 4. Summary of organ weights in 4-week study

\begin{tabular}{lcccc}
\hline Dose (mg/kg) & 0 & 0.4 & 1.3 & 4.0 \\
\hline No. of Animals & 10 & 10 & 10 & 8 \\
Ovary (R) (mg) & $39 \pm 4.6$ & $40 \pm 6.1$ & $41 \pm 4.3$ & $36 \pm 6.8$ \\
Ovary (L) (mg) & $36 \pm 4.5$ & $37 \pm 3.5$ & $13 \pm 1.9$ & $38 \pm 5.4$ \\
Pituitary (mg) & $13 \pm 1.4$ & $13 \pm 3.2$ & $401 \pm 133.8$ & $13 \pm 1.9$ \\
Uterus (mg) & $486 \pm 104.6$ & $473 \pm 128.2$ & $463 \pm 45.2$ \\
\hline
\end{tabular}

$*: \mathrm{p}<0.05, * *: \mathrm{p}<0.01$ 
Repeated dose toxicity and fertility studies of indomethacin in rats

Table 5. Summary of pathological changes in the ovary in 2-week study (HE and anti-PCNA staining)

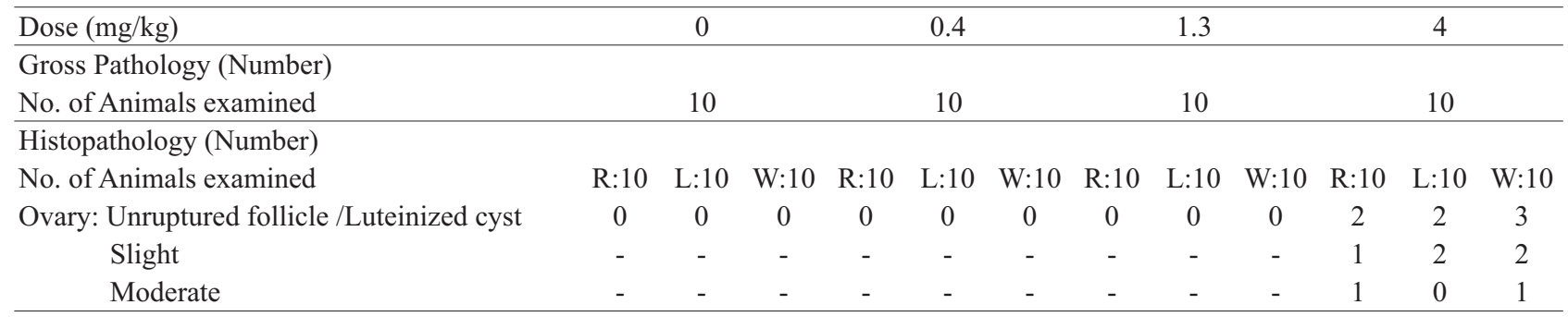

R: right ovary, L: left ovary, W: whole body, -: no noteworthy findings, Slight: Found in 2 follicles or less, Moderate: Found in 3 or 4 follicles or with antral dilatation, Severe: Found in 5 follicles or more.

Table 6. Summary of pathological changes in the ovary in 4-week study (HE and anti-PCNA staining)

\begin{tabular}{|c|c|c|c|c|c|c|c|c|c|c|c|c|}
\hline \multirow{2}{*}{$\frac{\text { Dose (mg/kg) }}{\text { Gross Pathology (Number) }}$} & \multicolumn{3}{|c|}{0} & \multicolumn{3}{|c|}{0.4} & \multicolumn{3}{|c|}{1.3} & \multicolumn{3}{|c|}{4} \\
\hline & & & & & & & & & & & & \\
\hline No. of Animals examined & & 10 & & & 10 & & & 10 & & & 10 & \\
\hline Histopathology (Number) & & & & & & & & & & & & \\
\hline No. of Animals examined & $\mathrm{R}: 10$ & L:10 & $\mathrm{W}: 10$ & $\mathrm{R}: 10$ & $\mathrm{~L}: 10$ & $\mathrm{~W}: 10$ & $\mathrm{R}: 10$ & $\mathrm{~L}: 10$ & $\mathrm{~W}: 10$ & $\mathrm{R}: 10$ & $\mathrm{~L}: 10$ & $\mathrm{~W}: 10$ \\
\hline Ovary: Unruptured follicle & 0 & 0 & 0 & 0 & 0 & 0 & 0 & 0 & 0 & 2 & 1 & 2 \\
\hline Slight & - & - & - & - & - & - & - & - & - & 2 & 1 & 2 \\
\hline Ovary: Follicular Cyst & 0 & 0 & 0 & 0 & 0 & 0 & 0 & 0 & 0 & 1 & 1 & 1 \\
\hline Moderate & - & - & - & - & - & - & - & - & - & 1 & 1 & 1 \\
\hline
\end{tabular}

R: right ovary, L: left ovary, W: whole body, -: no noteworthy findings, Slight: Found in 2 follicles or less, Moderate: Found in 3 or 4 follicles or with antral dilatation, Severe: Found in 5 follicles or more.

adrenals were found. Many of these changes indicate anemia or peritonitis or are secondary to inflammation due to gastrointestinal ulcer caused by indomethacin. There were no notable changes in the $1.3 \mathrm{mg} / \mathrm{kg}$ and $0.4 \mathrm{mg} / \mathrm{kg}$ groups in either study.

\section{Female fertility study}

In the fertility study, 8 rats given $4 \mathrm{mg} / \mathrm{kg}$ of indomethacin showed decreased spontaneous motility, moist fur around the urethral orifice, a prone position, and soiled fur around the nose. These animals then died or were sacrificed moribund due to gastrointestinal damage, as was the case in the 4-week toxicity study.

The mean body weight in the $4 \mathrm{mg} / \mathrm{kg}$ group was lower than that of the control throughout the dosing period.

Three animals in the $4 \mathrm{mg} / \mathrm{kg}$ group had more than 3 consecutive days of prolonged diestrus in the period before mating. This finding was not thought to be a direct effect of indomethacin, but rather due to poor condition exacerbated by gastrointestinal damage.

During the 2 -week mating period, no significant differences in the copulation index, fertility index or mean number of days prior to copulation were noted between the control group and any test article-treated group.

The number of corpora lutea, implantations, embryo- fetal deaths, live fetuses, and pre- and post-implantation losses (\%) in the surviving animals dosed with 0.4 , and $1.3 \mathrm{mg} / \mathrm{kg}$ were not significantly different from those in the control. Numeric values at $4 \mathrm{mg} / \mathrm{kg}$ were not analyzed statistically because of the high death rate (Table 7).

\section{Discussion}

In the general toxicity study, the treatment increased unruptured follicle or luteinized cysts in 3 and 2 animals in the $4 \mathrm{mg} / \mathrm{kg}$ groups in the 2- and 4-week studies, respectively. One animal was in the metestrus phase, and the others were in the estrus phase. An ovum was seen in the antrum of the cyst in the animal in the metestrus phase and one of those in the estrus phase. Indomethacin is an inhibitor of prostaglandin synthesis and blocks ovulation in rats (Sato et al., 1974). Shirota et al. (1998) reported that a single intravenous injection of $500 \mu \mathrm{g}$ indomethacin with 10 IU human chorionic gonadotropin induced unruptured luteinized follicles in the ovaries of rats without causing enteric lesions. They also reported that the same changes could not be effectively induced at the higher dose, which was associated with enteric lesions. The changes found in the present studies may not be secondary to ulcers or peritonitis. In the present study, the results that the ova were seen in an unruptured folli- 

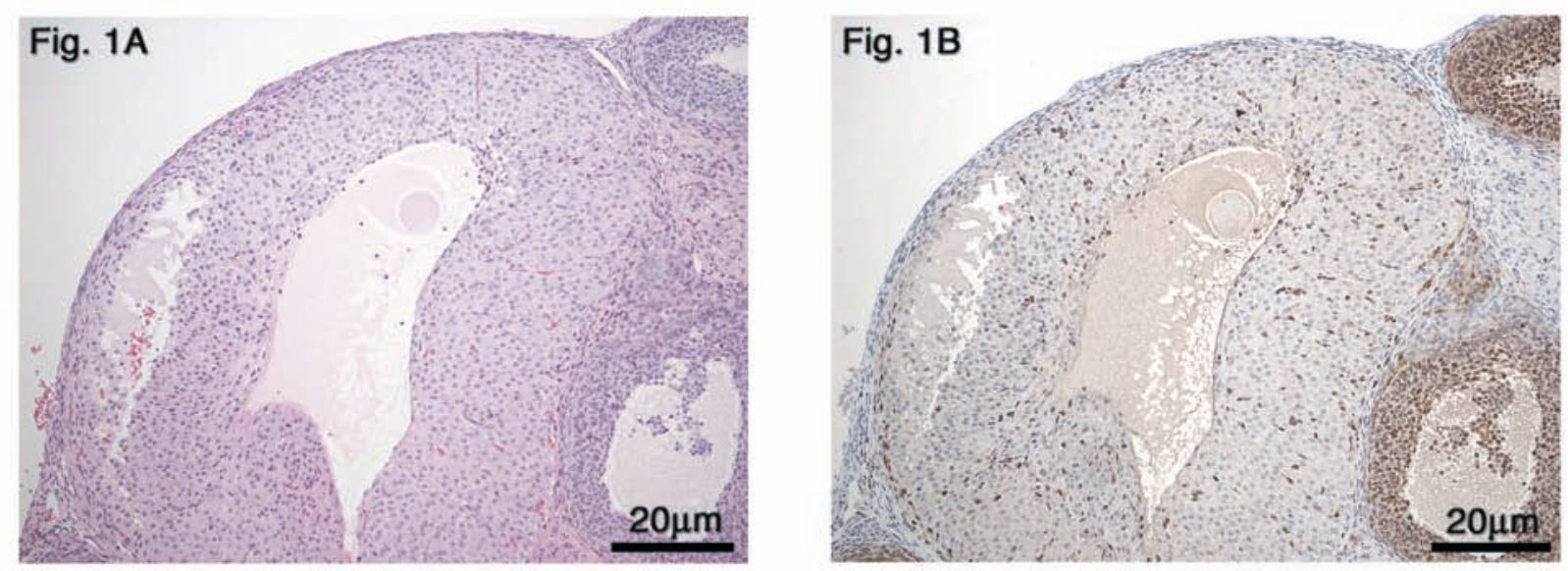

Fig. 1. A luteinized cyst observed in the $4 \mathrm{mg} / \mathrm{kg}$ group of the 2 -week study. The animal was in the metestrus phase and the luteal cells were completely similar to normal ones at this stage. An ovum with zona pellucida was observed in the cavity, indicating this corpus luteum was unruptured. A: HE staining, B: anti-PCNA immunohistochemical staining.

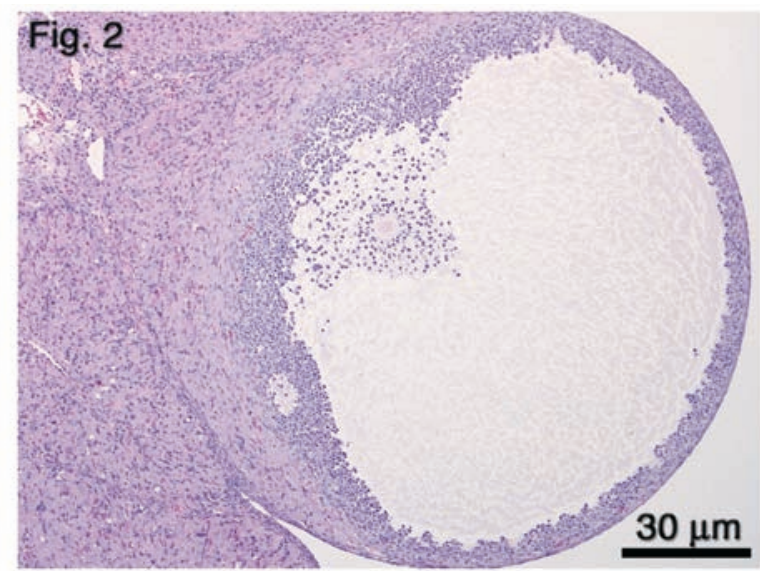

Fig. 2. An unruptured follicle observed in the $4 \mathrm{mg} / \mathrm{kg}$ group of the 4-week study. The animal was in the estrus phase. The follicle was cystic and contained an ovum in the antrum. Cumuli cells were expanded around the ovum. (HE)

cle and a luteinized cyst demonstrate inhibition of follicle rupture by indomethacin treatment, being in line with the previous report (Shirota et al., 1998).

Although follicular cysts were observed in only one animal in the $4 \mathrm{mg} / \mathrm{kg}$ group of the 4 -week study, it was thought to be a drug-induced change since its diameter was larger than that of normal corpora lutea and 3 or 4 follicular cysts were observed in each side of ovary from this animal. The follicular epithelium was thin, and apoptotic figures were more prominent than in the normal atresias of other animals in the metestrus phase. There was no notable change in the ovary, uterus, vagina or pituitary of the 2 animals that died in the 4-week study.

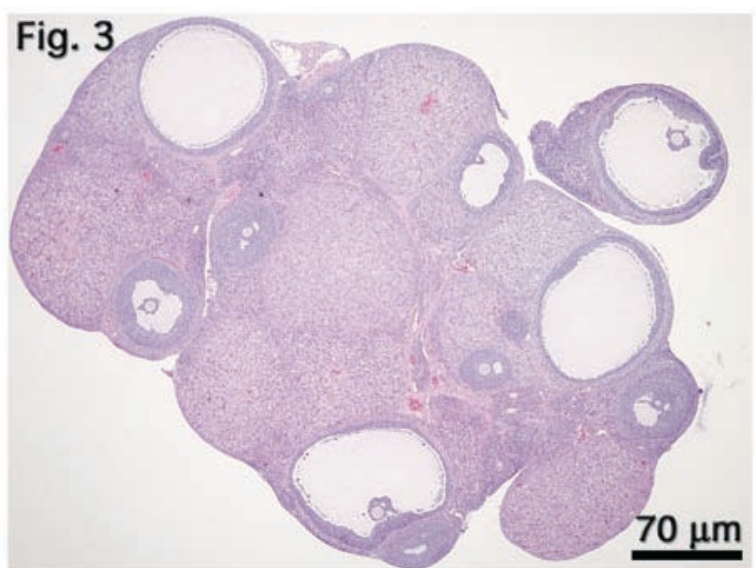

Fig. 3. Follicular cysts observed in the $4 \mathrm{mg} / \mathrm{kg}$ group of the 4-week study. The animal was in the diestrus phase. In these cystic follicles, cell debris in the antrum are prominent. (HE)

Anti-PCNA immunohistochemical staining was used to distinguish follicles from the background (Muskhelishvili et al., 2005), and aided the evaluation of the HE-stained specimens in the present studies, although there were no notable changes obtained only from anti-PCNA immunohistchemically stained specimens.

In the fertility study, 8 of 10 animals at the $4 \mathrm{mg} / \mathrm{kg}$ group died or were sacrificed moribund, and no significant difference in the tested items was detected between any dosing group and the control. Therefore, $1.3 \mathrm{mg} / \mathrm{kg}$ was considered to be no observed adverse effect level. Six animals died within the 2 -week pre-mating period, and 2 died during the mating period. All 8 of these animals 
Table 7. Summary of female fertility study

\begin{tabular}{lcccc}
\hline Dose (mg/kg/day) & 0 (Control) & 0.4 & 1.3 & 4.0 \\
\hline No. of Animals (Female) & 10 & 10 & 10 & 10 \\
No. that Died or were Sacrificed moribund & 0 & 0 & 0 & 8 \\
Clinical Observations & - & - & - & $1)$ \\
Body Weight Gain (g) (Days 1-15) & 19 & 20 & 14 & $5 * *$ \\
Mean No. Estrus phase/(15 days) & 3.7 & 3.8 & 3.7 & 3.8 \\
Irregular Estrous Cycle 2) & 0 & 1 & 2 & 3 \\
No. Animals Mated & 10 & 10 & 10 & 3 \\
No. Animals Copulated & 10 & 10 & 10 & 3 \\
No. Pregnant Animals & 10 & - & - & 2 \\
Necropsy Findings & - & 15.8 & 14.4 & $3)$ \\
Mean No. Corpora Lutea & 14.9 & 14.6 & 13.4 & 12.5 \\
Mean No. Implantations & 14.0 & 7.1 & 7.2 & 10.0 \\
Mean \% Preimplantation Loss & 5.5 & 13.9 & 12.4 & 19.8 \\
Mean No. Live Embryo & 13.1 & 0.7 & 1.0 & 9.5 \\
Mean No. Dead Embryo & 0.9 & 4.9 & 7.4 & 0.5 \\
Mean \% Postimplantation Loss & 6.5 & \multicolumn{1}{c}{. } & 5.6 \\
\hline
\end{tabular}

1):Decreased spontaneous motility, moist fur around urethral orifice, prone position, soiled fur around nose.

2) Irregular estrous cycle means that the diestrus phase persists for 3 consecutive days or longer.

3):Adhesion of various internal celiac organs.

- : No noteworthy findings, ${ }^{*}: \mathrm{p}<0.05, * *: \mathrm{p}<0.01$

had intestinal ulcers and peritonitis, which seemed to be more severe in the fertility study than the general toxicity studies. The animals were 10 weeks-old in the fertility study and 6 weeks-old in the 2- and 4-week studies, when these studies started. Aging reportedly increases the risk of NSAID-induced reactions (Johnson and Day, 1991); therefore, since the conditions and dosages were the same in these studies, the difference in sensitivity observed may have been caused by an age-dependent factor.

The morphological features of the female reproductive organs change every day according to estrous cycle phase (Organization for Economic Co-operation and Development, 2008; Westwood, 2008). Taking these changes into account may be a great help to histopathological observation. For example, in the estrus phase, large cystic follicles showing signs of luteinization can be categorized as unruptured follicles, even if any ova are not detected. Unruptured follicles observed at estrus stage are developing to luteinized cysts at normal metestrus in normal cycling rats. In the normal metestrus phase, however, it may be difficult to distinguish luteinized cysts with advanced luteinization from a cystic corpora lutea formed after regular ovulation (Kennedy and Miller, 1993) if an ovum is not present on the specimen. Therefore, for precise histopathological examination of the female reproductive organs, it is desirable to evaluate each phase so that each particular change can be delineated.
In conclusion, detailed histopathological observation with particular attention to the estrous cycle allowed for the detection of indomethacin-induced ovarian toxicity in both the 2- and 4-week repeated dose toxicity studies. The frequency of unruptured follicle in the 2-week study did not differ from that in the 4-week study, although no follicular cyst was found in the 2 -week study. This result suggests that a 2-week period is sufficient to detect ovarian toxicity.

\section{REFERENCES}

Armstrong, D.T. and Grinwich, D.L. (1972): Blockade of spontaneous and LH-induced ovulation in rats. Prostaglandins, 1, 21-28.

Brogden, R.N. (1986): Non-steroidal anti-inflammatory analgesics other than salicylates. Drugs, 32 Suppl. 4, 27-45.

Dunnett, C.W. (1964): New tables for multiple comparison with a control. Biometrics, 20, 482-491.

Japanese Association for Laboratory Animal Science. (1987): Guideline for Animal experimentation. Exp. Anim., 36, 285-288.

Johnson, A.G. and Day R.O. (1991): The problems and pitfalls of NSAID therapy in the elderly (Part I). Drugs Aging, 1, 130-143.

Kennedy, P.C. and Miller, R.B. (1993): The female genital system. In Pathology of domestic animals fourth edition (Jubb, K.V.F., Kennedy, P.C. and Palmer, N. eds.), pp. 349-470, Academic Press, San Diego.

Khan, K.N., Alden, C.L., Gleissner, S.E., Gessford, M.K. and Maziasz, T.J. (1998): Effect of papillotoxic agents on expression of cyclooxygenase isoforms in the rat kidney. Toxicol. Pathol., 26, 137-142. 


\section{K. Tsubota et al.}

Muskhelishvili, L., Wingard, S.K. and Latendresse, J.R. (2005): Proliferating cell nuclear antigen-A marker for ovarian follicle counts. Toxicol. Pathol., 33, 365-368.

Organization for Economic Co-operation and Development (2008): Part 3: Female Reproductive System. In Endocrine Disruption: A guidance document for histologic evaluation of endocrine and reproductive tests, Draft 3. Available from: http://www.oecd.org/ document/12/0,3343,en_2649_34365_1898188_1_1_1_1,00. html.

Satoh, H., Inada, I., Hirata, T. and Maki, Y. (1981): Indomethacin produces gastric antral ulcers in the refed rat. Gastroenterology., $\mathbf{8 1}, 719-25$.
Sato, T., Taya, K., Jyujo, T. and Igarashi, M. (1974): Ovulation block by indomethacin, an inhibitor of prostaglandin synthesis: A study of its site of action in rats. J. Reprod. Fertil., 39, 33-40.

Shirota, M., Watanabe, G., Taya, K. and Sasamoto, S. (1998): Effects of indomethacin on the selective release of follicle-stimulating hormone during the period of ovulation in the rat. J. Vet. Med. Sci., 60, 1059-1065.

Tsafriri, A., Lindner, H.R., Zor, U. and Lamprecht, S.A. (1972): Physiological role of prostaglandins in the induction of ovulation. Prostaglandins, 2, 1-10.

Westwood, F.R. (2008): The female rat reproductive cycle: A practical histological guide to staging. Toxicol. Pathol., 36, 375-384. 